\title{
RESEARCH
}

Open Access

\section{Metformin induces apoptosis in mesenchymal stromal cells and dampens their therapeutic efficacy in infarcted myocardium}

Xiao He ${ }^{1,2}$, Meng-Wei Yao ${ }^{1,2,3}$, Ming Zhu ${ }^{1,2}$, Dong-Lan Liang ${ }^{1,2,4}$, Wei Guo ${ }^{1,2}$, Yi Yang ${ }^{1,2}$, Rong-Seng Zhao ${ }^{1,2}$, Ting-Ting Ren ${ }^{1,2,4}$, Xiang Ao ${ }^{1,2}$, Wei Wang ${ }^{5}$, Chun-Yu Zeng ${ }^{5}$, Hua-Ping Liang ${ }^{2}$, Dong-po Jiang ${ }^{6}$, Jian $Y_{u^{7}}$ and Xiang $X u^{1,2,3^{*}}$ (i)

\begin{abstract}
Background: Cardiovascular complications, especially myocardial infarctions (MIs), are the leading mortality cause in diabetic patients. The transplantation of stem cells into damaged hearts has had considerable success as a treatment for $\mathrm{Ml}$, although whether antidiabetic drugs affect the therapeutic efficacy of stem cell transplantation is still unknown. This study aims to understand whether and how metformin, one of the first-line drugs used to treat type 2 diabetes mellitus ( $\mathrm{T}_{2} \mathrm{DM}$ ), induces mesenchymal stromal cell (MSC) apoptosis and dampens their cardioprotective effect after transplantation into infarcted hearts.

Methods: A mouse MI model was generated via permanent ligation of the left anterior descending (LAD) coronary artery. MSCs with or without metformin treatment were transplanted after MI in diabetic mice. Echocardiography was used to assess cardiac function and determine cardiac remodeling, and TTC staining was performed to evaluate infarction size. A mouse gavage model was performed to evaluate bone marrow MSCs for flow cytometry assay.

Results: Metformin dampened MSC therapeutic efficacy, which increased infarct size and restricted functional cardiac recovery. Specifically, metformin induced the activation of AMP-activated protein kinase (AMPK)-mediated apoptosis through the inhibition of S6K1-Bad-BCl-xL cell survival signaling, resulting in the upregulated expression of apoptosisassociated proteins and increased MSC apoptosis. Accordingly, counteracting AMPK attenuated metformin-induced apoptosis in MSCs and partially restored their cardioprotective effects in diabetic mice with MI. Furthermore, a decrease in peripheral blood MSCs was found in patients with $\mathrm{T}_{2} \mathrm{DM}$ who had a metformin medication history.
\end{abstract}

Conclusions: Our results highlight an unexpected adverse effect of metformin-induced MSC apoptosis through AMPKmediated mTOR suppression, which is attenuated by an AMPK inhibitor. Moreover, AMPK inhibition may be a novel strategy for enhancing the effectiveness of stem cell therapy after Ml in diabetes.

Keywords: Mesenchymal stromal cells, Metformin, Myocardial infarction, Apoptosis, Diabetes mellitus

\footnotetext{
* Correspondence: xiangxu@tmmu.edu.cn

'Department of Stem Cell and Regenerative Medicine, State Key Laboratory

of Trauma, Burn and Combined Injury, Daping Hospital and Research Institute of Surgery, Army Medical University, Chongqing, People's Republic of China

${ }^{2}$ First Department, State Key Laboratory of Trauma, Burn and Combined Injury, Daping Hospital and Research Institute of Surgery, Army Medical University, Chongqing, People's Republic of China

Full list of author information is available at the end of the article
}

(c) The Author(s). 2018 Open Access This article is distributed under the terms of the Creative Commons Attribution 4.0 International License (http://creativecommons.org/licenses/by/4.0/), which permits unrestricted use, distribution, and reproduction in any medium, provided you give appropriate credit to the original author(s) and the source, provide a link to the Creative Commons license, and indicate if changes were made. The Creative Commons Public Domain Dedication waiver (http://creativecommons.org/publicdomain/zero/1.0/) applies to the data made available in this article, unless otherwise stated. 


\section{Background}

Cardiovascular complications, especially myocardial infarction (MI), are the leading cause of death in diabetic patients [1, 2]. Recent studies reported that injected stem cells can salvage the myocardium from death through a protective paracrine mechanism and that several types of stem cells have been tested for safety and efficacy in animal models of MI and human MI patients [3, 4].

Mesenchymal stromal cells (MSCs), which contain a subpopulation of stem cells able to differentiate in bone, cartilage, and fat, are one of the most promising candidates for stem cell therapy for heart disease. MSCs exist in most tissues in the body including the umbilical cord, bone marrow, adipose tissue, and peripheral blood; exhibit a strong capacity for replication in vitro; express CD105, CD90, and CD73; express low levels of MHC-I; and lack expression of MHC-II, CD11b, CD14, CD34, CD45, and CD31 [5]. The isolation of MSCs according to the International Society for Cellular Therapy (ISCT) criteria has produced heterogeneous, nonclonal cultures of stromal cells containing stem cells with different multipotent properties, committed progenitors, and differentiated cells [6]. MSCs in situ are speculated to have an indispensable role in tissue homeostasis by replacing dysfunctional or dead cells [7]. Recently, our group found that the number of circulating mesenchymal-like cells (CD34-/CD105+) is significantly decreased in type 2 diabetes mellitus $\left(\mathrm{T}_{2} \mathrm{DM}\right)$ patients and is negatively correlated with the progression of chronic diabetic complications [8]. In addition, our group reported that the transplantation of MSCs into damaged hearts has a therapeutic effect on MI and that prolyl hydroxylase domain protein 2 (PHD2) silencing could enhance the protective effect of MSCs on cardiomyocytes [9]. Since cardiovascular complications, especially MI, greatly rely on MSCs to regenerate cardiac tissue and promote a functional recovery, it is of great importance to determine whether antidiabetic drugs impact the therapeutic efficacy of stem cell transplantation in patients with diabetes mellitus. Moreover, because diabetic patients are chronically exposed to antidiabetic drugs, this long-term exposure and drug accumulation resulting from the compromised renal and hepatic functions commonly seen in these patients may amplify the toxic effects of antidiabetic drugs [10]. Therefore, understanding the effects of antidiabetic agents on stem cell biology is indispensable for the development of stem cell-based therapy for treating diabetic patients with MI.

Few studies have investigated the effects that antidiabetic drugs have on stem cells. Some have suggested that antidiabetic agents affect the multipotency and proliferation of MSCs. Benvenuti et al. and Beck et al. reported that thiazolidinediones (TZDs) stimulate adipogenesis and decrease osteoblastogenesis in human MSCs [11, 12]. On the other hand, insulin can promote the proliferation and osteogenic differentiation of human MSCs [13]. In addition, metformin has previously been shown to promote the osteogenic differentiation and inhibit the adipogenic differentiation of MSCs [14]. However, little is known about the effect of antidiabetic agents on the therapeutic efficacy of stem cell transplantation in diabetic patients with MI. To study the effects of antidiabetic drugs on the therapeutic efficacy of stem cell transplantation, we screened common clinical antidiabetic agents such as various insulin analogs, TZDs, and metformin in vitro. Specifically, we assessed the proliferation and apoptosis of MSCs after treatment with these drugs. We discovered that metformin, which is recommended by the American Diabetes Association (ADA) as a first-line hypoglycemic treatment for $\mathrm{T}_{2} \mathrm{DM}$, clearly induced MSC apoptosis. To clarify the correlation between metformin and stem cell transplantation and to reveal the mechanism underlying our preliminary observations, we further performed a series of in vitro and in vivo experiments. Here, we report that metformin dampens MSC therapeutic efficacy, which increased infarct size and restricted functional cardiac recovery, through inducing MSC apoptosis in an AMPK-mTOR pathway-dependent manner. Additionally, AMPK inhibition may be a novel strategy for enhancing the effectiveness of stem cell therapy after MI in diabetes.

\section{Methods}

\section{Human samples}

Blood samples were obtained from the Hypertension and Endocrinology Department of the Third Affiliated Hospital, Third Military Medical University, after Ethics Committee approval based on informed consent from patients. All participants gave written consent before participation in the study. The investigation conforms to the principles outlined in the Declaration of Helsinki. Upon arrival in the laboratory, the samples were immediately processed for the detection of hMSCs by flow cytometry, as described in the protocol (hMSC Analysis Kit, BD Stemflow, USA).

\section{Cells}

Human umbilical cords $(n=5)$ were collected from full-term women immediately after cesarean section in the Gynaecology Department of the Third Affiliated Hospital, Army Military Medical University of China, after approval was obtained from the Institutional Ethics Committee. After vessel removal, the cords were cut into pieces with scissors and cultured in $\alpha$-MEM $(10 \%$ FBS, $2 \mathrm{mM} \mathrm{L}$-glutamine). All MSCs used in the experiment were derived from passages 5-10. The MSCs were used after thorough characterization by flow cytometry 
(hMSC Analysis Kit, BD Stemflow, USA), as defined by the International Society for Cellular Therapy. After incubation in differentiation media, the MSCs differentiated into osteocytic, chondrocytic, and adipocytic lineages (Additional file 1: Figure S1).

\section{Cell apoptosis analysis}

To quantify the apoptotic MSCs, cells were harvested at $24 \mathrm{~h}, 48 \mathrm{~h}$, and $72 \mathrm{~h}$ after treatment with different concentrations of metformin with or without compound $\mathrm{C}$ $(1 \mu \mathrm{M})$ and siAMPK in $\alpha$-MEM $(10 \%$ FBS, $2 \mathrm{mM}$ L-glutamine). To quantify apoptosis, cells were washed and stained with an annexin V-FITC and propidium iodide (PI) apoptosis kit (KeyGen Biotech, China) according to the manufacturer's instructions. Stained cells were analyzed by flow cytometry (NovoCyteTM, ACEA Biosciences, USA), and the data were analyzed using NovoExpress V.1.2.1 software.

\section{Cell cycle analysis}

Cell cycle analyses were performed using Cell Cycle Analysis Kits (KeyGen Biotech, China) according to the manufacturer's instructions. A flow cytometer (NovoCyteTM, ACEA Biosciences, USA) was used to analyze cells and to determine the percentage of cells that were in the G0/G1, S, and G2/M phases of the cell cycle using NovoExpress V.1.2.1 software.

\section{Western blot}

MSCs were lysed in ice-cold lysis buffer on ice. A protein quantification of cell lysates was completed using a Bradford assay (Bio-Rad, Hercules, CA). Equal quantities of protein were separated by $8-12 \%$ SDS-PAGE and transferred to a nitrocellulose membrane. The membranes were blocked with 5\% nonfat milk in TBST solution (0.05\% Tween 20 in Tris-buffered saline) and then incubated overnight at $4{ }^{\circ} \mathrm{C}$ with primary antibodies. The blots were washed with TBST, incubated with the appropriate horseradish peroxidase-conjugated secondary antibodies (1:1000) for $2 \mathrm{~h}$ at room temperature, washed again with TBST, and then developed with ECL western blotting substrate (Thermo Scientific, Waltham, MA). The following antibodies were purchased from CST: AMPK, phospho-AMPK, Akt, phospho-S473-Akt, phospho-T308-Akt, mTOR, phospho-mTOR, S6K1, phospho-S6K1, RagB, LC3B, Bad, phospho-Bad, Bcl-xL, cleaved-caspase 3 , and $\beta$-actin.

\section{Mice}

A genetic mouse model of diabetes B6.BKS(D)-Lepr ${ }^{\mathrm{db}} / \mathrm{J}$ (Jackson Laboratory, Bar Harbor, ME, USA) was bred in the Experimental Animal Center of Third Affiliated Hospital, Third Military Medical University; the detailed methods for generating the diabetic myocardial infarction mouse model followed those described in our previous study [8]. The optimal oral metformin dosage for many diabetic patients is $\sim 2 \mathrm{~g} /$ day. The human metformin dosage of $20 \mathrm{mg} / \mathrm{kg} /$ day orally translates to an equivalent mouse dosage of $250 \mathrm{mg} / \mathrm{kg} / \mathrm{day}$, based on the normalization to body surface area [15]. In this experiment, the 8- to 12-week-old male diabetic mice were treated with saline, metformin $(250 \mathrm{mg} / \mathrm{kg} /$ day, i.g., Sigma), or metformin + compound C $(0.1 \mathrm{mg} / \mathrm{kg} / \mathrm{day}$, i.g., Sigma) decoction by gavage for 4 weeks. Afterward, all the diabetic mice were sacrificed to isolate bone marrow MSCs for flow cytometry assay. A permanent ligation of the left anterior descending (LAD) coronary artery and cell injection were performed in male diabetic mice ( 8 weeks old). Immediately after coronary ligation, $1 \times 10^{5}$ MSCs suspended in $50 \mu \mathrm{L}$ PBS were injected at five sites in the anterior and posterior infarct border zones of the ischemic myocardium. FK506 (3 mg/kg/ day, i.p., Sigma) was administered to MSC-transplanted and sham-operated animals daily from 1 week before the operation until the end of the study. Metformin $(250 \mathrm{mg} / \mathrm{kg} /$ day, 4 weeks, i.g., Sigma) or metformin + compound C $(0.1 \mathrm{mg} / \mathrm{kg} /$ day, 4 weeks, i.g., Sigma) was administered to LAD-operated animals daily from the day of operation until 4 weeks after operation. Animal care and all experimental procedures were performed in strict accordance with the approved protocols and animal welfare regulations of the Animal Care and Use Committee at the Third Military Medical University and EU (Directive 2010/63/EU) ethical guidelines and comply with the National Institutes of Health guide for the care and use of laboratory animals. Echocardiography was used to assess the cardiac function and determine cardiac remodeling. TTC staining was performed to evaluate infarction size.

\section{Real-time cell proliferation monitoring}

MSCs were seeded at densities of $5 \times 10^{3}$ cells/well into an E-plate 16 (ACEA Biosciences, San Diego, CA) containing $100 \mu \mathrm{L}$ medium per well and monitored on an xCELLigence Real-Time Cell Analyzer Dual Plate (RTCA DP) instrument (ACEA Biosciences). When the cells entered the log phase, $2 \mathrm{mM}$ metformin, $2 \mathrm{mM}$ metformin $+1 \mu \mathrm{M}$ compound $\mathrm{C}$, or $2 \mathrm{mM}$ metformin + siAMPK was added in $\alpha$-MEM $(10 \%$ FBS, $2 \mathrm{mM}$ $\mathrm{L}$-glutamine). The cells were treated for $160 \mathrm{~h}$ and incubated at $37{ }^{\circ} \mathrm{C}$ in a $5 \% \mathrm{CO}_{2}$ atmosphere. RTCA software v. 1.2.1 was used to record the cell index (CI). All experiments were repeated at least three times.

\section{Flow cytometry (FCM) analysis}

For live cell counting, transplanted MSCs were labeled with CM-DiI (CellTracker ${ }^{\mathrm{ra}}$ CM-DiI Dye, Invitrogen, Thermo Fisher, USA) according to the manufacturer's 
instructions, and hearts with MI were digested. Dissociated cells were separated by filtering through a $30-\mu \mathrm{m}$ filter. Most of the cardiomyocytes ( $>30 \mu \mathrm{m}$ diameters) were discarded, and the small cell fraction $(<30 \mathrm{um})$ was collected. The surviving CM-DiI (PE+) cells were identified with fluorescence microscopy, and the number of CM-DiI (PE+) cells was counted with NovoExpress V.1.2.1 software.

\section{Statistical analysis}

Unless specified otherwise, all experiments were repeated at least three times. Data are presented as the means \pm SD. Statistical analysis (ANOVA and Student's $t$ test) was performed using SPSS 13.0 software; $p<0.05$ was considered to indicate statistical significance.

\section{Results}

\section{Metformin dampens MSC therapeutic efficacy in MI}

To determine whether taking metformin impairs the therapeutic effect of stem cells, we evaluated the effects of MSC transplantation on infarct size and cardiac function in post-MI diabetic mouse hearts [9]. Myocardial infarct size was significantly smaller in diabetic mice treated with MSCs than in those treated with PBS or metformin at 4 weeks post-MI, whereas the metformin + MSCs group showed that metformin dampens the MSC therapeutic efficacy in MI (Fig. 1a, b). Although the infarction size in the metformin group was larger than that in the PBS group, there was no significant difference $(p=0.2453)$. These data suggest that the regeneration of cardiac tissue after MSC transplantation was limited by metformin treatment. In line with the histologic change, the left ventricle ejection fraction (EF) and fractional shortening (FS) of the post-MI hearts at 4 weeks were lower in diabetic mice treated with metformin + MSCs than in those treated with MSCs alone (Fig. 1c, e, and f). MI significantly decreased the EF and FS in all groups of post-MI hearts compared to sham-operated hearts (Fig. 1e, f). Left ventricle dilation induced by MI injury was confirmed with diastolic left ventricle internal diameter (LVIDd) of post-MI hearts at 4 weeks, which was significantly larger than that of the sham group. However, MSC but not MSC + metformin transplantation significantly limited the increase in LVIDd induced by MI (Fig. 1g). These results indicate that MSC transplantation reduces the post-MI deterioration of

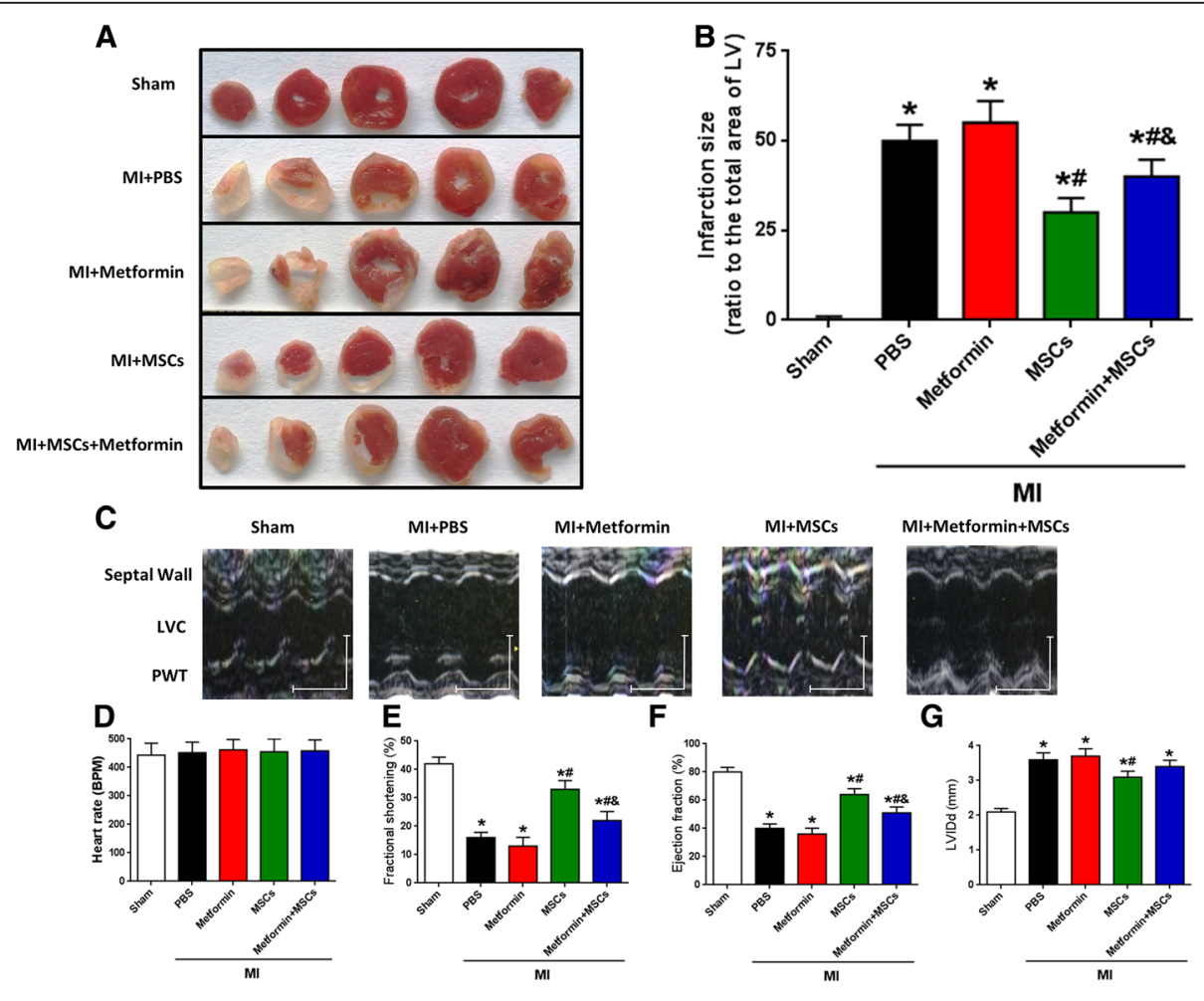

Fig. 1 Metformin dampens MSC therapeutic efficacy in MI. a Representative images of TTC-stained heart sections obtained from Sham, MI + PBS, $\mathrm{Ml}+$ metformin, $\mathrm{Ml}+\mathrm{MSC}$, and $\mathrm{Ml}+$ metformin + MSCs groups at 4 weeks after MI. $\mathbf{b}$ LV infarct sizes expressed as the ratio of the area of the infarct band to the total area of LV $(n=23)$. c Representative M-mode images of hearts with sham surgery or Ml at 4 weeks after PBS, metformin, MSCs, and metformin + MSCs treatment. Scale bar: $X$ axis: $0.1 \mathrm{~s} ; Y$ axis: $0.2 \mathrm{~cm}$. $\mathbf{d}$ Heart rates were controlled to be similar in different groups $(n=23)$. $\mathbf{e}-\mathbf{g}$ LV fraction shortening (e), LV ejection fraction $(\mathbf{f})$, and diastolic left ventricle internal diameter (LVIDd) (g) at 4 weeks after treatment $(n=23) .{ }^{*} p<0.05$ vs. sham; ${ }^{\#} p<0.05$ vs. Ml + metformin; ${ }^{~} p<0.05$ vs. Ml + MSCs, by one-way ANOVA 
cardiac function and that this cardioprotective effect is impaired by metformin treatment in diabetic mice.

\section{Metformin induces MSC apoptosis}

To directly test whether endogenous MSCs were affected by metformin in the animal model, we evaluated the CD45-/CD105+/CD29+/Sca-1+ cells in the bone marrow of diabetic mice treated with saline or metformin $(250 \mathrm{mg} / \mathrm{kg} /$ day $)$ decoction by gavage for 4 weeks. After treatment with saline or metformin $(250 \mathrm{mg} / \mathrm{kg} /$ day) decoction by gavage for 4 weeks, the diabetic mice were sacrificed to isolate the bone marrow for the flow cytometry assay (Fig. 2a). As expected, metformin treatment led to a decrease in the number of diabetic mouse bone marrow MSCs (CD45-/CD105+/CD29+/Sca-1+) (Fig. 2b, c). Together, these results show that metformin caused a decrease in the endogenous MSC levels in diabetic mice.

To understand how metformin decreases the levels of MSCs, we monitored apoptosis, autophagy, and proliferation inhibition. Given that most current in vitro studies of metformin drug concentration use mM levels [16], which are far greater than the physiological dose [17], we decided to start from the $\mu \mathrm{M}$-class drug concentration, which is close to the physiological dose, in the following experiments. Neither autophagy nor proliferation were affected by treatment, but metformin greatly increased the rate of apoptosis (annexin $\mathrm{V}+$ ) of human umbilical cord-derived mesenchymal stromal cells (hUC-MSCs) in vitro in a dose- (100 $\mu \mathrm{M}, 250 \mu \mathrm{M}$, $500 \mu \mathrm{M}, 1 \mathrm{mM}, 2 \mathrm{mM})$ and time-dependent manner (Fig. 2d, e), suggesting that metformin induces apoptosis

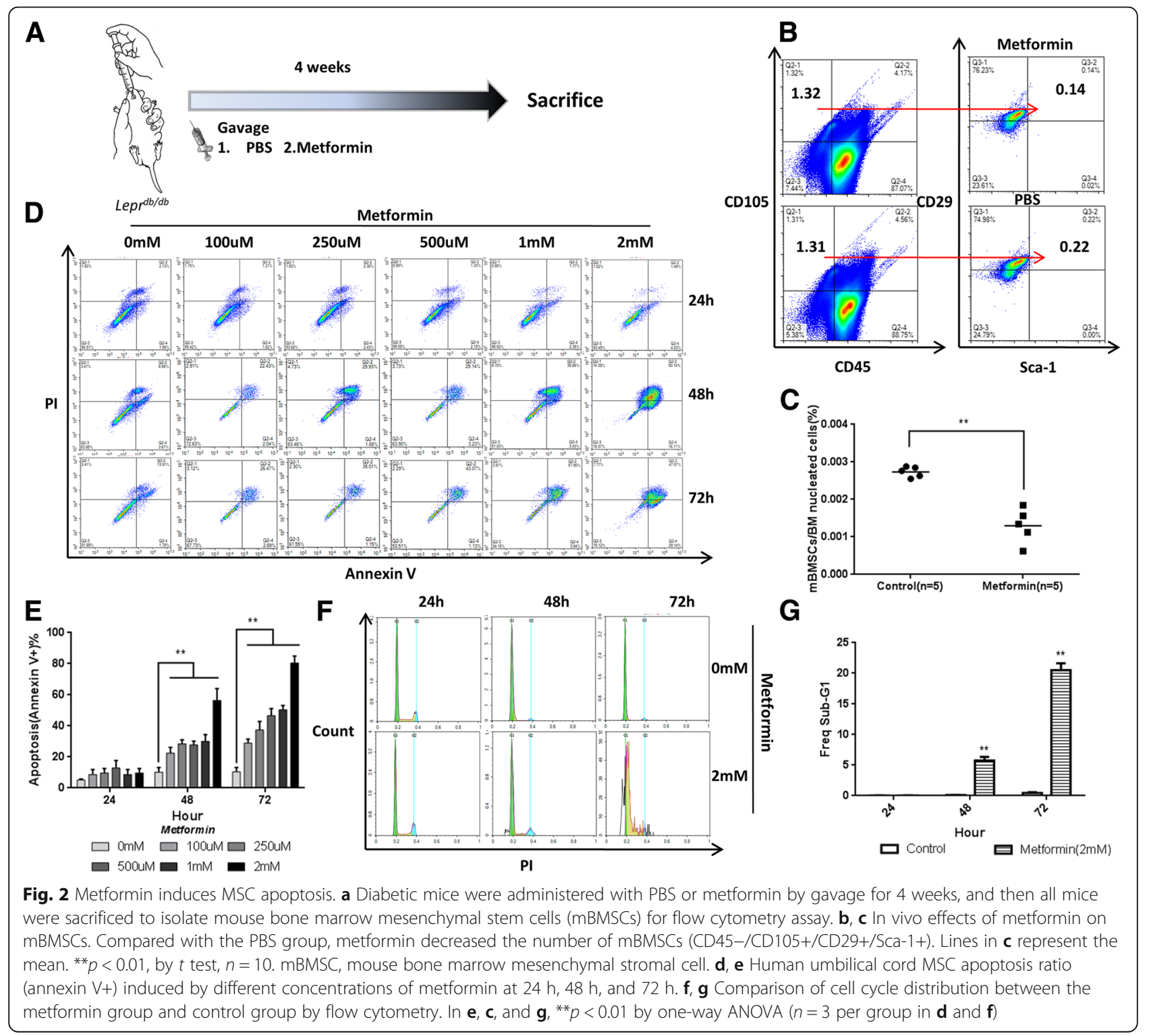


in hUC-MSCs. Incubation with $2.0 \mathrm{mM}$ metformin for $48 \mathrm{~h}$ or $72 \mathrm{~h}$ increased the rate of apoptosis to $71.3 \%$ or $89.2 \%$, respectively.

In addition to MSCs, there are cardiomyocytes and myocardial blood vessels that could affect the recovery of cardiac function after MI. Thus, we studied the effects of metformin on mouse cardiomyocytes and human endothelial cells. However, metformin had no such effect on cardiomyocytes and endothelial cells (Additional file 1: Figure S2A-S2D). Moreover, metformin also had no such effect on adult cells such as fibroblasts (Additional file 1: Figure S2E and S2F).

To quantify the magnitude of metformin-induced apoptosis in MSCs, we studied the effects of metformin on cell cycle distribution and progression. As shown in Fig. 2c, d, compared to the control, metformin induced a sub-G1 peak of approximately $20 \%$ at $72 \mathrm{~h}$, which is suggestive of apoptosis.

\section{Metformin induces the activation of AMP-activated protein kinase (AMPK)-mediated apoptosis}

Metformin has previously been shown to inhibit the growth of breast cancer cell lines in vitro via AMPK induction and mTOR inhibition [18]. To determine whether a similar mechanism is involved in metformin-induced MSC apoptosis, we explored the AMPK-mTOR signaling pathway. As shown in Fig. 3a, metformin treatment activated AMPK and suppressed mTOR and its downstream effector S6K1, a signaling pathway critical for cell proliferation and survival. Furthermore, the metformin-induced hUC-MSC apoptosis was confirmed by the increased levels of cleaved-caspase 3 (Fig. 3a).

Next, we investigated whether a specific inhibitor of AMPK, compound C, and specific siAMPK (Fig. 3f, g) reverse the inhibitory effects of metformin on MSC survival. As shown in Fig. 3b, c, treatment with compound C $(1 \mu \mathrm{M})$ and siAMPK significantly inhibited metformin-induced MSC apoptosis, AMPK activation, and mTOR and S6K1 suppression (Fig. 3e). In support of this evidence, real-time cellular analysis (RTCA) also showed decreased metformin-induced apoptosis in hUC-MSCs when treated with compound C and siAMPK for $160 \mathrm{~h}$ (Fig. 3d).

In addition to AMPK, the mTOR pathway is also regulated by Akt, which belongs to a family of lipid kinases critical for cell growth, survival, and proliferation [19]. To explore which pathway is the main regulator of mTOR, we explored the Akt- and AMPK-mTOR signaling pathways. As shown in Fig. 3e, treatment of MSCs with an AMPK inhibitor reversed metformin-activated AMPK, which suppressed mTOR and its downstream effector S6K1. However, no significant change in Akt was observed.

In addition to apoptosis-related caspase 3, autophagy -related LC3B was also considered. However, the level of
LC3B was not significantly different between groups (Fig. 3e), and metformin-induced MSC apoptosis was not changed after treatment with an autophagy inhibitor (3-methyladenine, 3-MA). In addition, metformin treatment did not change the level of Rag B, which was reported to be involved in mTOR inhibition by metformin [20].

S6K1 signals cell survival and cell growth through the phosphorylation of substrates such as the Bcl-2 family member Bad and the ribosomal subunit S6, respectively $[21,22]$. Treatment with metformin significantly reduced Bad phosphorylation (Fig. 3h). Prior studies have established that Bad phosphorylation coordinates mitochondrial energy metabolism and apoptosis [22] and that nonphosphorylated Bad heterodimerizes with $\mathrm{Bcl}-\mathrm{xL}$ or $\mathrm{Bcl}-2$ at the mitochondrial membrane to promote cell death [23]. Figure 3h shows that phosphorylation of Bad decreased its binding with Bcl-xL; however, the binding of Bad with Bcl-xL increased dramatically with an increase in apoptosis but only in the metformin group. Taken together, these results suggest that metformin induces MSC apoptosis in an AMPK-mTOR-S6k1-Bad-dependent manner.

\section{Counteraction of AMPK partially restored MSC cardioprotective effects in diabetic mice with MI}

To verify whether AMPK inhibition could protect hearts with myocardial infarction from metformin-induced MSC apoptosis in vivo, we used a myocardial infarction model in diabetic mice to evaluate the infarct size and cardiac function in post-MI hearts. Myocardial infarct size was significantly larger in diabetic mice treated with metformin + MSCs than in those treated with MSCs at 4 weeks post-MI, whereas the metformin + MSCs + compound $\mathrm{C}$ group showed a partially restored MSC therapeutic efficacy in MI (Fig. 4a, b). In line with the histologic change, the left ventricle EF and FS of the post-MI hearts at 4 weeks were lower in the diabetic mice treated with metformin + MSCs than in those treated with metformin + MSCs + compound C (Fig. 4e, f). In addition, MSC and metformin + compound C + MSC but not metformin + MSC transplantation significantly limited the increase in LVIDd induced by MI (Fig. 4g). These results indicate that MSC transplantation reduces the post-MI deterioration of cardiac function; this cardioprotective effect is impaired by metformin treatment in diabetic mice, and this adverse effect can be attenuated by counteraction of AMPK in diabetic mice.

\section{Counteraction of AMPK attenuated metformin-induced MSC apoptosis in vivo}

The in vitro data suggest that AMPK inhibition can prevent metformin-induced MSC apoptosis. Is it possible that AMPK inhibition can prevent metformin-induced MSC apoptosis in vivo? To test this hypothesis, we set up an in vivo experiment by treating diabetic mice with 


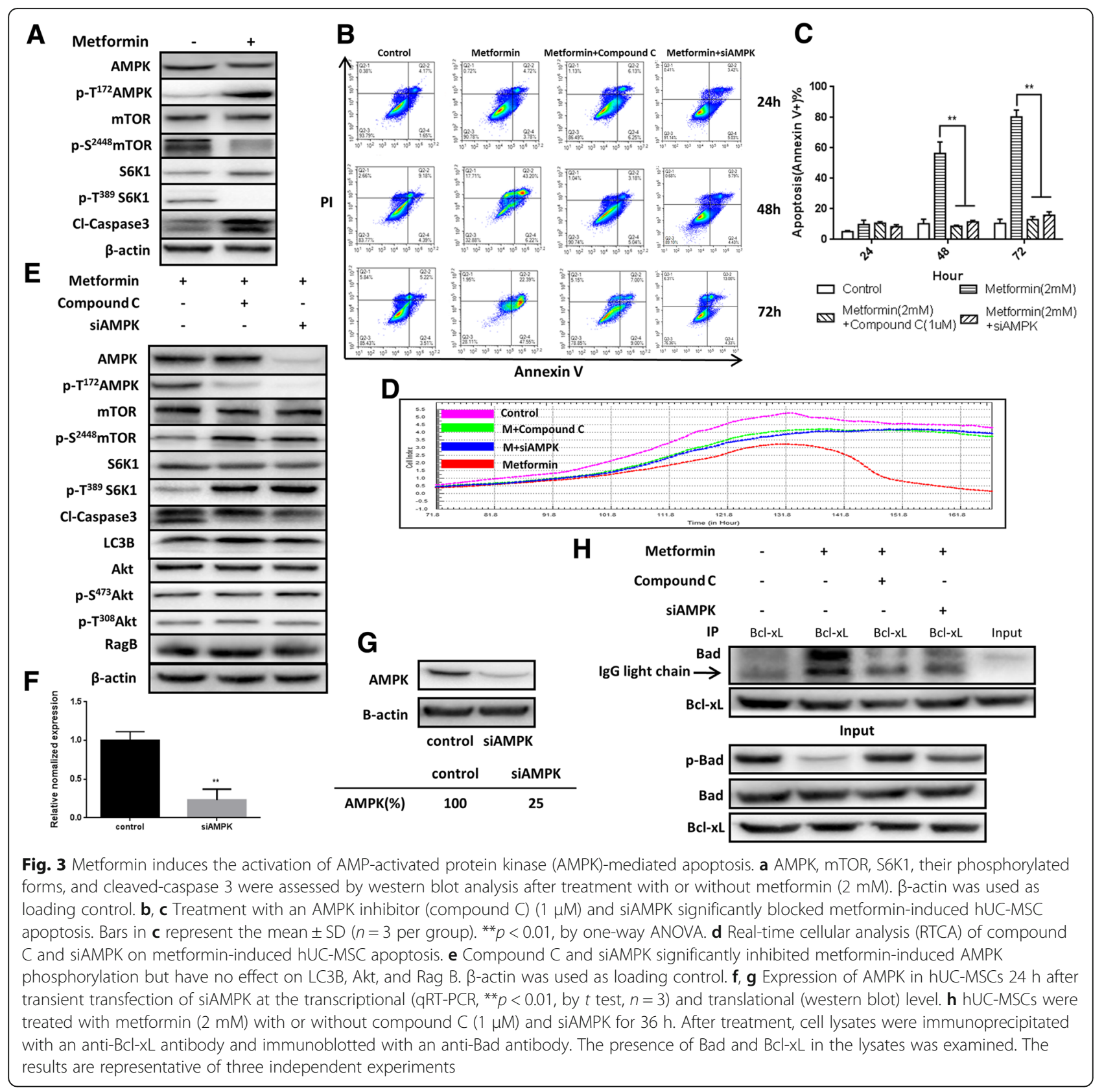

either metformin or metformin with compound C. After treatment with PBS, metformin $(250 \mathrm{mg} / \mathrm{kg} /$ day $)$, or metformin + compound C $(0.1 \mathrm{mg} / \mathrm{kg} /$ day $)$ decoction by gavage for 4 weeks, metformin treatment was shown to induce a significant decrease in diabetic mouse bone marrow MSCs compared with that from PBS treatment. As expected, compared with metformin alone, compound $\mathrm{C}$ impaired the metformin-induced mouse bone marrow MSC decrease (CD45-/CD105+/CD90+/Sca-1+) (Fig. 5a, b).

To further confirm that metformin induces MSC apoptosis in vivo, the survival of transplanted CM-DiI-labeled MSCs in MI hearts was quantified. MI hearts were digested at $4 \mathrm{~h}, 48 \mathrm{~h}$, and 7 days post-transplantation. There were significantly less CM-DiI-labeled cells in the myocardium in the MSCs + metformin group than in the MSCs group at 7 days after transplantation; however, compound $\mathrm{C}$ reversed this effect in the MSCs + metformin + compound C group (Fig 5c, d). The better survival rate of MSCs in the MSCs and MSCs + metformin + compound C groups was confirmed with FCM analysis of isolated CM-DiI $(\mathrm{PE}+)$ cells at multiple time points post-transplantation (Fig. 5e).

\section{Metformin may display negative effects on endogenous MSCs in diabetic patients}

To further characterize the effect of metformin on endogenous MSCs, we recruited $10 \mathrm{~T}_{2} \mathrm{DM}$ patients without 


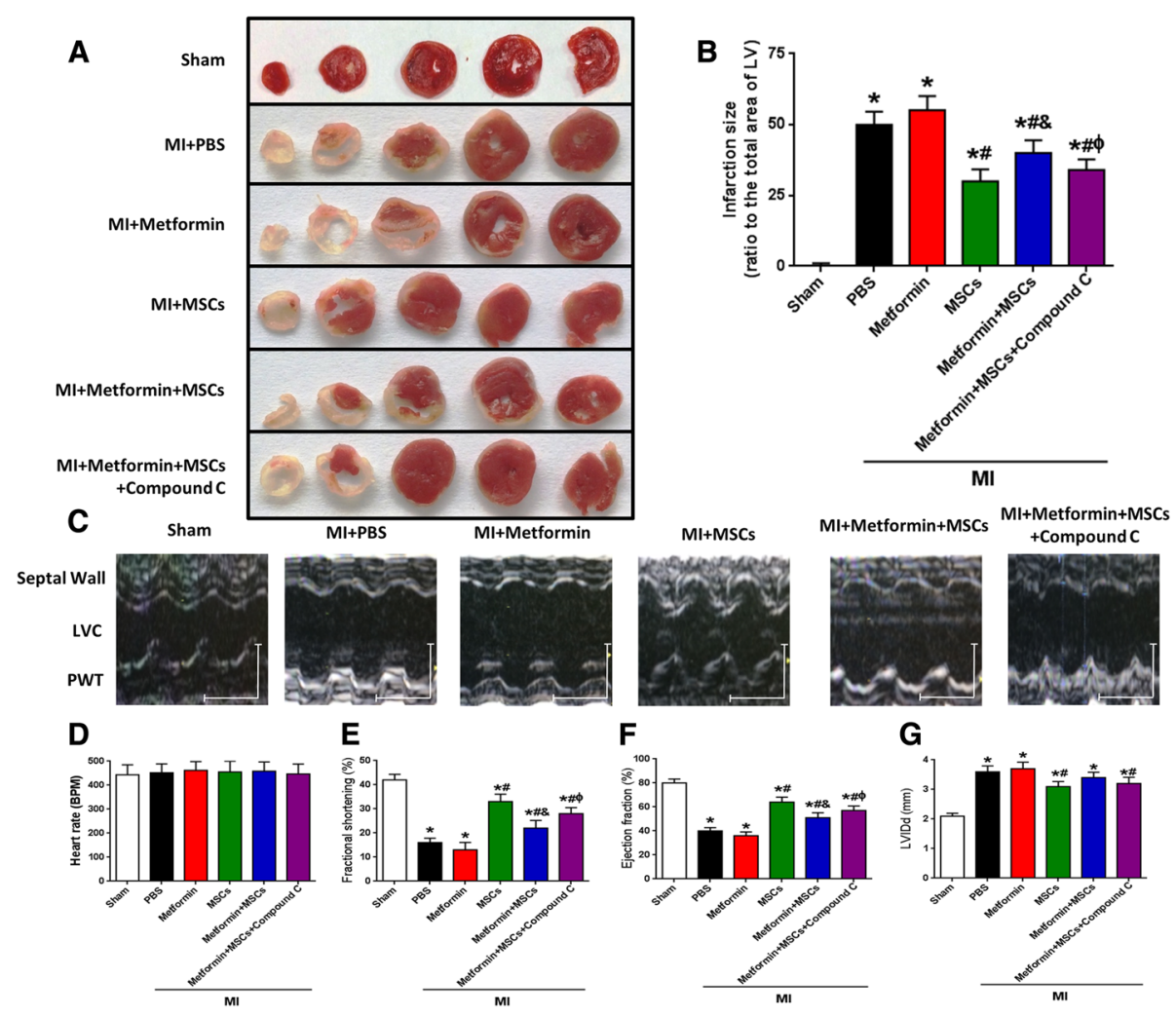

Fig. 4 Counteraction of AMPK partially restored MSC cardioprotective effects in diabetic mice with MI. a Representative images of TTC-stained heart sections obtained from sham, Ml + PBS, MI + metformin, MI + MSCs, Ml + metformin + MSCs, and Ml + metformin + MSCs + compound C groups at 4 weeks after MI. $\mathbf{b}$ LV infarct sizes expressed as the ratio of the area of the infarct band to the total area of LV $(n=28)$. $\mathbf{c}$ Representative M-mode images of hearts with sham surgery or Ml at 4 weeks after PBS, metformin, MSCs, metformin + MSCs, and metformin + MSCs + compound C treatment. Scale bar: $X$ axis: 0.1 s; $Y$ axis: $0.2 \mathrm{~cm}$. $\mathbf{d}$ Heart rates were controlled to be similar in different groups. e- $\mathbf{g}$ LV fraction shortening $(\mathbf{e})$, LV ejection fraction ( $\mathbf{f}$ ), and diastolic left ventricle internal diameter (LVIDd, $\mathbf{g})$ at 4 weeks after treatment $(n=28) .{ }^{*} p<0.05$ vs. sham; ${ }^{*} p<0.05$ vs. Ml + metformin; ${ }^{\circledR} p<0.05$ vs. Ml + MSCs; $^{\phi} p<0.05$ vs. Ml + metformin + MSCs, by one-way ANOVA

metformin medication history $\left(\mathrm{T}_{2} \mathrm{DM}, n=10\right), 10 \mathrm{~T}_{2} \mathrm{DM}$ patients with metformin medication history $\left(\mathrm{T}_{2} \mathrm{DM}-\mathrm{M}\right)$, and 10 healthy volunteers (Additional file 1: Table S1) to quantify the number of peripheral blood MSCs (CD34 -/CD11b-/CD19-/CD45-/HLA-DR-/CD90+/CD73 $+/ \mathrm{CD} 105+)$. The mean counts of MSCs in peripheral blood of $\mathrm{T}_{2} \mathrm{DM}\left(297.8 \pm 64.42 / 10^{\wedge} 6\right.$ cells, $\left.n=10\right)$ and $\mathrm{T}_{2} \mathrm{DM}-\mathrm{M}\left(239.7 \pm 49.08 / 10^{\wedge} 6\right.$ cells, $\left.n=10\right)$ patients were significantly lower than those of healthy volunteers (395.2 $\pm 75.61 / 10^{\wedge} 6$ cells, $\left.n=10\right)(p=0.0057$ and $p<0.0001$, respectively) (Fig. 5f, g). Although the number of peripheral blood MSCs in the $\mathrm{T}_{2} \mathrm{DM}-\mathrm{M}$ group was lower than that in the $\mathrm{T}_{2} \mathrm{DM}$ group, there was no significant difference $(p=$ 0.1241 ). This result suggests that metformin may negatively impact endogenous MSCs in diabetic patients.

\section{Discussion}

The major cause of death and complications in $\mathrm{T}_{2} \mathrm{DM}$ patients is cardiovascular disease. More than $60 \%$ of all $\mathrm{T}_{2} \mathrm{DM}$ patients die of cardiovascular complications and an even greater percentage have serious complications.
MSC-based cell therapy not only is applicable to MI but also has an antidiabetic effect in patients with diabetes $[9,24]$. In addition, with the progress of aging, senescence abrogates the therapeutic potential of MSCs, so it is more necessary to use exogenous MSCs for treatment [25]. Therefore, we can foresee that in the near future, MSC-based cell therapy will be more attractive in the clinic.

MSCs are multipotent active cells with immunoregulation and tissue-repair capacities. These cells are located in virtually all postnatal organs and tissues [26]. Upon tissue damage, resident MSCs rapidly function as seed cells to help recruit a large number of MSCs from peripheral circulation to the injury site to participate in repair and regeneration $[27,28]$. The efficiency of MSCs in tissue repair depends on their quality and quantity. Many studies have shown that impaired MSC quality plays a pathogenic role in diabetes [29-33], in addition to reduced numbers of MSCs [34, 35]. Therefore, we must determine not only the endogenous disease factors resulting in MSC reduction and/or dysfunction but also 

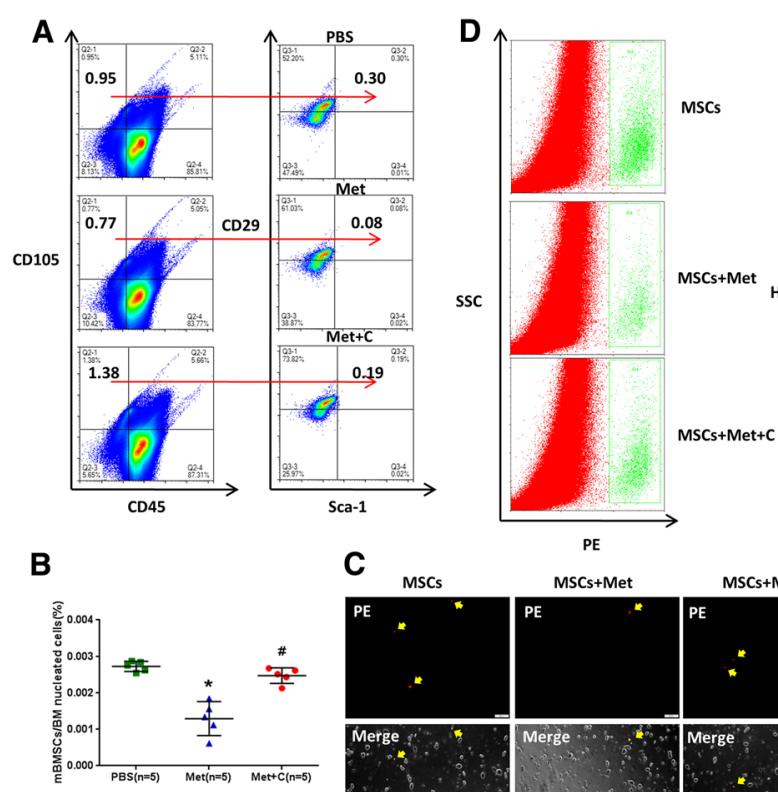

C Mscs
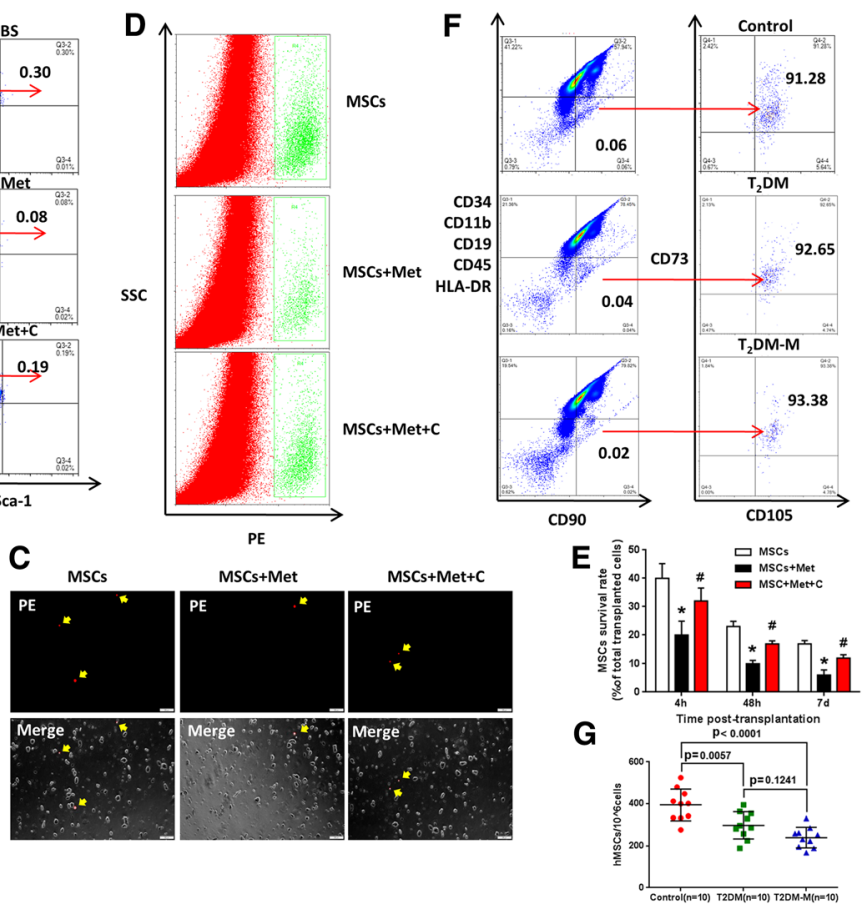

Fig. 5 Counteraction of AMPK attenuated metformin-induced MSC apoptosis in vivo. a Diabetic mice were administered with PBS, metformin (250 mg/kg/day, i.g.), or metformin + compound C ( $0.1 \mathrm{mg} / \mathrm{kg} /$ day, i.g.) by gavage for 4 weeks, and then all mice were sacrificed to isolate mBMSCs for flow cytometry assay. b Metformin treatment induced a significant decrease in mBMSCs compared with PBS treatment. Compared with metformin, compound $C$ reduced the metformin-induced mBMSC decrease (CD45-/CD105+/CD90+/Sca-1+). ${ }^{*} p<0.01 \mathrm{vs.} \mathrm{PBS,}{ }^{*} p<0.01 \mathrm{vs}$. Met, by one-way ANOVA, $n=5$ per group. c Post-MI hearts with CM-Dil-labeled MSC transplantation were enzymatically digested, and small cells from the heart ( $<30 \mu \mathrm{m}$ diameter) were collected after the depletion of cardiomyocytes. As indicated with a yellow arrow, CM-Dil-labeled cells represent surviving MSCs under fluorescence microscopy. Scale bar $=100 \mu \mathrm{m}$. d Representative flow cytometric plots of surviving CM-Dil+ MSCs counted by FCM. Gate R4 indicates the CM-Dil+ cells out of all the isolated cells from the heart. $\mathbf{e}$ The percentage of surviving MSCs out of the total transplanted MSCs at different time points. ${ }^{*} p<0.05$ vs. MSCs, ${ }^{\#} p<0.05$ vs. MSCs + Met, by one-way ANOVA, $n=15$ per time points. $\mathbf{f}, \mathbf{g}$ Comparison among human peripheral blood MSCs (CD34-/CD11b-/CD19-/CD45-/HLA-DR-/CD90+/CD73+/CD105+) from healthy controls (control, $n=10)$, diabetic patients without metformin medication history $\left(T_{2} D M, n=10\right)$, and diabetic patients with metformin medication history $\left(T_{2} \mathrm{DM}-\mathrm{M}, n=10\right)$. Symbols represent individual subjects; horizontal lines show the mean; and data are presented as the means $\pm S D$, statistical test applied by one-way ANOVA. Met metformin, $\mathrm{C}$ compound $\mathrm{C}_{1} \mathrm{~T}_{2} \mathrm{DM}$ type 2 diabetes mellitus, mBMSC mouse bone marrow mesenchymal stromal cell

the impact of exogenous factors on MSCs, including various drugs involved in the treatment of diseases.

MSCs have great treatment potential for use in ischemic heart disease $[9,36]$. However, a major challenge to MSC therapy is that transplanted cells undergo apoptosis. Recent studies have reported that endogenous disease factors such as myocardial infarction-related hypoxia and serum deprivation induced significant MSC apoptosis [37]. In addition, diabetes-enhanced TNF- $\alpha$ reduced MSC proliferation and increased MSC apoptosis [38]. Our group recently found that a decrease in circulating mesenchymal-like cells $(\mathrm{CD} 34+/ \mathrm{CD} 105+)$ is associated with the progression of chronic diabetic complications in $\mathrm{T}_{2} \mathrm{DM}$ [8]. Therefore, we speculate that in addition to endogenous factors including the activation of the complement system in diabetic patients recently reported by our group [8], exogenous factors, especially drugs, may have unexpected adverse effects that influence the quality and quantity of MSCs.
Many in vitro and in vivo studies have demonstrated the growth-inhibiting effects of metformin in breast, lung, endometrial, liver, medullary thyroid, and gastric cancer cell lines [39]. Several cohort studies have reported a correlation between metformin and improved cancer survival [40-43]. Moreover, metformin shows a cytotoxic effect on cancer stem cells [16]. Therefore, compared with the common cell culture additive insulin, metformin is more likely to have adverse effects on adult stem cells such as MSCs. As expected, the hypoglycemic drug used in $\mathrm{T}_{2} \mathrm{DM}$, metformin, was found to dampen the MSC transplantation therapeutic effect, resulting in the impaired regeneration and repair of target tissues including the heart in an MI diabetic mouse model.

We further hypothesized that the negative effect of metformin on the therapeutic efficacy of exogenous transplanted MSCs in MI is related to MSC apoptosis. After treatment with metformin, the apoptosis of MSCs was found both in 
vitro and in vivo but with little effect on cardiomyocytes, endothelial cells, and fibroblasts. Specifically, metformin induces activation of AMPK-mediated apoptosis through inhibition of S6K1-Bad-Bcl-xL cell survival signaling, resulting in the upregulated expression of apoptosis-associated proteins and increased MSC apoptosis. Accordingly, the counteraction of AMPK via the AMPK inhibitor compound $\mathrm{C}$ or siAMPK attenuated metformin-induced apoptosis in MSCs and partially restored their cardioprotective effects in diabetic mice with MI.

Except for apoptosis, metformin may also induce senescence which has a big impact on the biology of cells. Senescence has been associated with telomere shortening and dysfunction, mesenchymal progenitor cell dysfunction, cytokine production changes, and a reduced capacity of bone marrow stromal cells to maintain functional hematopoeitic stem cells [44]. Besides, Alessio et al. reported that low-dose radiation induced senescence of human mesenchymal stromal cells and impaired the autophagy process [45]. Specifically, increased expression of pigment epithelium-derived factor in aged mesenchymal stem cells impairs their therapeutic efficacy for attenuating myocardial infarction injury [46]. Further studies should study whether metformin influences MSC's senescence, thereby affecting its therapeutic role in the transplantation of myocardial infarction.

Although metformin caused a decrease in mouse bone marrow MSCs, metformin alone increased the infarct size of MI mouse model with no significant difference. We speculated that the possible reason is that the MSCs of the mouse itself mobilized to the site of myocardial infarction are far less than our exogenously transplanted MSCs during a short time. Furthermore, decreased peripheral blood MSCs were found in $\mathrm{T}_{2} \mathrm{DM}$ patients with a metformin medication history. Because of the small sample size and the small proportion of patients with $\mathrm{T}_{2} \mathrm{DM}$ who had no metformin medication history, there was no significant difference in the number of peripheral blood MSCs between the $\mathrm{T}_{2} \mathrm{DM}-\mathrm{M}$ and $\mathrm{T}_{2} \mathrm{DM}$ groups.

\section{Conclusions}

Our results highlight an unexpected adverse effect of metformin-induced MSC apoptosis through AMPK-mediated mTOR suppression that is attenuated by AMPK inhibition. Metformin was confirmed to induce apoptosis in exogenous MSCs and then affect their therapeutic efficacy. In addition, the results from animals and humans suggest that metformin has a negative effect on endogenous MSCs. Lastly, maximizing the therapeutic effects of MSC-based therapy in diabetes mellitus while minimizing the adverse effects of metformin on MSCs may be possible. Several new strategies can be considered, including taking metformin with an AMPK inhibitor, not taking metformin during MSC transplantation, or developing a modified metformin with reduced adverse effects on MSCs.

\section{Additional file}

\begin{abstract}
Additional file 1: Table S1. Demographic and clinical characteristics of patients. Figure S1. Identification of MSCs. A hUC-MSC in normal condition (40X); B osteogenic differentiation of hUC-MSC; C chondrogenic differentiation of hUC-MSC; D adipogenic differentiation of hUC-MSC; E detection of surface markers of hUC-MSC by flow cytometry. Figure S2. Metformin has no effect on mouse cardiomyocytes, human endothelial cells, and human fibroblast survival. A and $\mathrm{B}$, the ratio of mouse cardiomyocyte apoptosis (annexin $\mathrm{V}+$ ) induced by $2 \mathrm{mM}$ metformin at $24 \mathrm{~h}, 48 \mathrm{~h}$, and $72 \mathrm{~h} ; \mathrm{C}$ and D, the ratio of human endothelial cells apoptosis (annexin $\mathrm{V}_{+}$) induced by $2 \mathrm{mM}$ metformin at $24 \mathrm{~h}, 48 \mathrm{~h}$, and $72 \mathrm{~h} ; \mathrm{E}$ and $\mathrm{F}$, the ratio of human fibroblast apoptosis (annexin $\mathrm{V}_{+}$) induced by $2 \mathrm{mM}$ metformin at $24 \mathrm{~h}, 48 \mathrm{~h}$, and $72 \mathrm{~h}$. Bars in B, D, and $\mathrm{F}$ represent the mean $\pm \mathrm{SEM}$ ( $n=3$ per group). Statistical test applied by one-way ANOVA. (DOCX 11562 kb)
\end{abstract}

\section{Abbreviations}

AMPK: AMP-activated protein kinase; hUC-MSCs: Human umbilical cordderived mesenchymal stromal cells; LAD: Left anterior descending; Mls: Myocardial infarctions; MSCs: Mesenchymal stromal cells; $T_{2} D M$ : Type 2 diabetes mellitus

\section{Acknowledgements}

Not applicable.

\section{Funding}

This study was supported by the National Nature Science Foundation of China (NSFC, No. 81372027), the China Postdoctoral Science Foundation Funded Project (No. 2015 M582842), and the Nature Scientific Foundation of Chongqing (No. cstc2015shmszx120108).

\section{Availability of data and materials}

The datasets supporting the results of this article are included within the article.

\section{Authors' contributions}

$X H$ and $X X$ designed the study. $X H, M Y, M Z, D L, W G$, and $Y Y$ performed the in vitro experiments. $X H, M Y, R Z, T R$, and $X A$ performed the in vivo experiments. WW and CZ performed and analyzed the echocardiography and $T T C$ experiments. $H L, D J, J Y$, and $X X$ reviewed the paper. $X H$ and $X X$ wrote the manuscript. All authors read and approved the final manuscript.

Ethics approval and consent to participate

The study was approved by the ethics committee of Daping Hospital, Army Medical University of the Chinese People's Liberation Army. A written informed consent was provided in accordance with the Declaration of Helsinki.

Consent for publication

Not applicable.

\section{Competing interests}

The authors declare that they have no competing interests.

\section{Publisher's Note}

Springer Nature remains neutral with regard to jurisdictional claims in published maps and institutional affiliations.

\section{Author details}

${ }^{1}$ Department of Stem Cell and Regenerative Medicine, State Key Laboratory of Trauma, Burn and Combined Injury, Daping Hospital and Research Institute of Surgery, Army Medical University, Chongqing, People's Republic of China. ${ }^{2}$ First Department, State Key Laboratory of Trauma, Burn and Combined Injury, Daping Hospital and Research Institute of Surgery, Army Medical University, Chongqing, People's Republic of China. ${ }^{3}$ Department of 
Biochemistry and Molecular Biology, College of Basic Medical Sciences, Army Medical University, Chongqing, People's Republic of China. ${ }^{4}$ Department of Histology and Embryology, Qingdao University Medical College, Qingdao, Shandong, People's Republic of China. ${ }^{5}$ Department of Cardiology, Daping Hospital and Research Institute of Surgery, Army Medical University, Chongqing, People's Republic of China. ${ }^{6}$ Department of Critical Care Medicine, Daping Hospital and Research Institute of Surgery, Army Medical University, Chongqing 400042, People's Republic of China. 'Department of Pathology of Pittsburgh Cancer Institute, Pittsburgh, PA, USA.

\section{Received: 29 August 2018 Revised: 17 October 2018} Accepted: 22 October 2018 Published online: 08 November 2018

\section{References}

1. Mangiapane H. Cardiovascular disease and diabetes. Adv Exp Med Biol. 2012;771:219-28.

2. Fox CS, Coady S, Sorlie PD, D'Agostino RB Sr, Pencina MJ, Vasan RS, Meigs JB, Levy D, Savage PJ. Increasing cardiovascular disease burden due to diabetes mellitus: the Framingham Heart Study. Circulation. 2007;115:154450 .

3. Ptaszek LM, Mansour M, Ruskin JN, Chien KR. Towards regenerative therapy for cardiac disease. Lancet. 2012;379:933-42.

4. Rasmussen TL, Raveendran G, Zhang J, Garry DJ. Getting to the heart of myocardial stem cells and cell therapy. Circulation. 2011;123:1771-9.

5. Dominici M, Le Blanc K, Mueller I, Slaper-Cortenbach I, Marini F, Krause D, Deans R, Keating A, Prockop D, Horwitz E. Minimal criteria for defining multipotent mesenchymal stromal cells. The International Society for Cellular Therapy position statement. Cytotherapy. 2006;8:315-7.

6. Squillaro T, Peluso G, Galderisi U. Clinical trials with mesenchymal stem cells: an update. Cell Transplant. 2016;25:829-48.

7. Hass R, Kasper C, Bohm S, Jacobs R. Different populations and sources of human mesenchymal stem cells (MSC): a comparison of adult and neonatal tissue-derived MSC. Cell Commun Signal. 2011;9:12.

8. Zhu M, He X, Wang XH, Qiu W, Xing W, Guo W, An TC, Ao LQ, Hu XT, Li Z, et al. Complement C5a induces mesenchymal stem cell apoptosis during the progression of chronic diabetic complications. Diabetologia. 2017;60(9): 1822-33.

9. Wang WE, Yang D, Li L, Wang W, Peng Y, Chen C, Chen P, Xia X, Wang H, Jiang J, et al. Prolyl hydroxylase domain protein 2 silencing enhances the survival and paracrine function of transplanted adipose-derived stem cells in infarcted myocardium. Circ Res. 2013;113:288-300.

10. Walker JJ, Johnson JA, Wild SH. Diabetes treatments and cancer risk: the importance of considering aspects of drug exposure. Lancet Diabetes Endocrinol. 2013;1:132-9.

11. Benvenuti S, Cellai I, Luciani P, Deledda C, Baglioni S, Giuliani C, Saccardi R, Mazzanti B, Dal Pozzo S, Mannucci E, et al. Rosiglitazone stimulates adipogenesis and decreases osteoblastogenesis in human mesenchymal stem cells. J Endocrinol Investig. 2007;30:RC26-30.

12. Beck GR Jr, Khazai NB, Bouloux GF, Camalier CE, Lin Y, Garneys LM, Siqueira $J$, Peng $L$, Pasquel $F$, Umpierrez $D$, et al. The effects of thiazolidinediones on human bone marrow stromal cell differentiation in vitro and in thiazolidinedione-treated patients with type 2 diabetes. Transl Res. 2013;161: 145-55.

13. Song-hao Z, Cheng-zhi $H, X u Y$, Yuan-he W, Shao-qi T, Kang S. Insulin promotes the osteogenic differentiation of umbilical cord mesenchymal stem cells. Chinese J Tissue Eng Res. 2016;20:807-12.

14. Gu Q, Gu Y, Yang H, Shi Q. Metformin enhances osteogenesis and suppresses adipogenesis of human chorionic villous mesenchymal stem cells. Tohoku J Exp Med. 2017:241:13-9.

15. Reagan-Shaw S, Nihal M, Ahmad N. Dose translation from animal to human studies revisited. FASEB J. 2008:22:659-61.

16. Wurth R, Pattarozzi A, Gatti M, Bajetto A, Corsaro A, Parodi A, Sirito R, Massollo M, Marini C, Zona G, et al. Metformin selectively affects human glioblastoma tumor-initiating cell viability: a role for metformin-induced inhibition of Akt. Cell Cycle. 2013;12:145-56.

17. Foretz M, Guigas B, Bertrand L, Pollak M, Viollet B. Metformin: from mechanisms of action to therapies. Cell Metab. 2014;20:953-66.

18. Zakikhani M, Dowling R, Fantus IG, Sonenberg N, Pollak M. Metformin is an AMP kinase-dependent growth inhibitor for breast cancer cells. Cancer Res. 2006;66:10269-73.
19. Liu P, Cheng H, Roberts TM, Zhao JJ. Targeting the phosphoinositide 3kinase pathway in cancer. Nat Rev Drug Discov. 2009;8:627-44.

20. Kalender A, Selvaraj A, Kim SY, Gulati P, Brule S, Viollet B, Kemp BE, Bardeesy $\mathrm{N}$, Dennis P, Schlager JJ, et al. Metformin, independent of AMPK, inhibits mTORC1 in a rag GTPase-dependent manner. Cell Metab. 2010;11:390-401.

21. Choo AY, Yoon SO, Kim SG, Roux PP, Blenis J. Rapamycin differentially inhibits S6Ks and 4E-BP1 to mediate cell-type-specific repression of mRNA translation. Proc Natl Acad Sci U S A. 2008;105:17414-9.

22. Danial NN. BAD: undertaker by night, candyman by day. Oncogene. 2008; 27(Suppl 1):S53-70.

23. Yang E, Zha J, Jockel J, Boise LH, Thompson CB, Korsmeyer SJ. Bad, a heterodimeric partner for $\mathrm{BCl}-\mathrm{XL}$ and $\mathrm{BCl}-2$, displaces $\mathrm{Bax}$ and promotes cell death. Cell. 1995:80:285-91.

24. Ezquer F, Ezquer M, Contador D, Ricca M, Simon V, Conget P. The antidiabetic effect of mesenchymal stem cells is unrelated to their transdifferentiation potential but to their capability to restore Th1/Th2 balance and to modify the pancreatic microenvironment. Stem Cells. 2012; 30:1664-74

25. Sepulveda JC, Tome M, Fernandez ME, Delgado M, Campisi J, Bernad A Gonzalez MA. Cell senescence abrogates the therapeutic potential of human mesenchymal stem cells in the lethal endotoxemia model. Stem Cells. 2014;32:1865-77.

26. Porada CD, Zanjani ED, Almeida-Porad G. Adult mesenchymal stem cells: a pluripotent population with multiple applications. Curr Stem Cell Res Ther 2006:1:365-9.

27. Wong SP, Rowley JE, Redpath AN, Tilman JD, Fellous TG, Johnson JR. Pericytes, mesenchymal stem cells and their contributions to tissue repair. Pharmacol Ther. 2015;151:107-20.

28. Dimarino AM, Caplan Al, Bonfield TL. Mesenchymal stem cells in tissue repair. Front Immunol. 2013:4:201.

29. Kim YS, Kwon JS, Hong MH, Kang WS, Jeong HY, Kang HJ, Jeong M, Ahn Y. Restoration of angiogenic capacity of diabetes-insulted mesenchymal stem cells by oxytocin. BMC Cell Biol. 2013;14:38.

30. Rodrigues M, Wong W, Rennert RC, Davis CR, Longaker MT, Gurtner GC. Progenitor cell dysfunctions underlie some diabetic complications. Am J Pathol. 2015:185:2607-18.

31. Yang G, Jia Y, Li C, Cheng Q, Yue W, Pei X. Hyperglycemic stress impairs the stemness capacity of kidney stem cells in rats. PLoS One. 2015;10:e0139607.

32. El-Ftesi S, Chang El, Longaker MT, Gurtner GC. Aging and diabetes impair the neovascular potential of adipose-derived stromal cells. Plast Reconstr Surg. 2009;123:475-85

33. Kim SM, Kim YH, Jun YJ, Yoo G, Rhie JW. The effect of diabetes on the wound healing potential of adipose-tissue derived stem cells. Int Wound J. 2016;13(Suppl 1):33-41.

34. Kondo M, Kamiya H, Himeno T, Naruse K, Nakashima E, Watarai A, Shibata T, Tosaki T, Kato J, Okawa T, et al. Therapeutic efficacy of bone marrowderived mononuclear cells in diabetic polyneuropathy is impaired with aging or diabetes. J Diabetes Investig. 2015;6:140-9.

35. Nowak WN, Borys S, Kusinska K, Bukowska-Strakova K, Witek P, Koblik T, Jozkowicz A, Malecki MT, Dulak J. Number of circulating pro-angiogenic cells, growth factor and anti-oxidative gene profiles might be altered in type 2 diabetes with and without diabetic foot syndrome. J Diabetes Investig. 2014:5:99-107.

36. Liu CB, Huang H, Sun P, Ma SZ, Liu AH, Xue J, Fu JH, Liang YQ, Liu B, Wu DY, et al. Human umbilical cord-derived mesenchymal stromal cells improve left ventricular function, perfusion, and remodeling in a porcine model of chronic myocardial ischemia. Stem Cells Transl Med. 2016;5:1004-13.

37. Li C, Guo Z, Guo B, Xie Y, Yang J, Wang A. Inhibition of the endogenous $\mathrm{CSE} / \mathrm{H}(2) \mathrm{S}$ system contributes to hypoxia and serum deprivation-induced apoptosis in mesenchymal stem cells. Mol Med Rep. 2014;9:2467-72.

38. Ko Kl, Coimbra LS, Tian C, Alblowi J, Kayal RA, Einhorn TA, Gerstenfeld LC, Pignolo $\mathrm{R}$, Graves DT. Diabetes reduces mesenchymal stem cells in fracture healing through a TNFalpha-mediated mechanism. Diabetologia. 2015;58:633-42.

39. Rizos CV, Elisaf MS. Metformin and cancer. Eur J Pharmacol. 2013;705:96108.

40. Landman GW, Kleefstra N, van Hateren KJ, Groenier KH, Gans RO, Bilo HJ. Metformin associated with lower cancer mortality in type 2 diabetes: ZODIAC-16. Diabetes Care. 2010:33:322-6.

41. Bowker SL, Yasui Y, Veugelers P, Johnson JA. Glucose-lowering agents and cancer mortality rates in type 2 diabetes: assessing effects of time-varying exposure. Diabetologia. 2010;53:1631-7. 
42. Currie $\mathrm{CJ}$, Poole CD, Jenkins-Jones S, Gale EA, Johnson JA, Morgan CL. Mortality after incident cancer in people with and without type 2 diabetes: impact of metformin on survival. Diabetes Care. 2012;35:299-304.

43. Franciosi M, Lucisano G, Lapice E, Strippoli GF, Pellegrini F, Nicolucci A. Metformin therapy and risk of cancer in patients with type 2 diabetes: systematic review. PLoS One. 2013:8:e71583.

44. Ju Z, Jiang H, Jaworski M, Rathinam C, Gompf A, Klein C, Trumpp A, Rudolph KL. Telomere dysfunction induces environmental alterations limiting hematopoietic stem cell function and engraftment. Nat Med. 2007; 13:742-7.

45. Alessio N, Del Gaudio S, Capasso S, Di Bernardo G, Cappabianca S, Cipollaro M, Peluso G, Galderisi U. Low dose radiation induced senescence of human mesenchymal stromal cells and impaired the autophagy process. Oncotarget. 2015;6:8155-66.

46. Liang H, Hou H, Yi W, Yang G, Gu C, Lau WB, Gao E, Ma X, Lu Z, Wei X, et al. Increased expression of pigment epithelium-derived factor in aged mesenchymal stem cells impairs their therapeutic efficacy for attenuating myocardial infarction injury. Eur Heart J. 2013;34:1681-90.

Ready to submit your research? Choose BMC and benefit from:

- fast, convenient online submission

- thorough peer review by experienced researchers in your field

- rapid publication on acceptance

- support for research data, including large and complex data types

- gold Open Access which fosters wider collaboration and increased citations

- maximum visibility for your research: over $100 \mathrm{M}$ website views per year

At $\mathrm{BMC}$, research is always in progress.

Learn more biomedcentral.com/submissions 